(C2007 IEEE. Personal use of this material is permitted. However, permission to reprint/republish this material for advertising or promotional purposes or for creating new collective works for resale or redistribution to servers or lists, or to reuse any copyrighted component of this work in other works must be obtained from the IEEE. 


\title{
Blind Signal Separation Using Steepest Descent Method
}

\author{
Hai Huyen Dam, Sven Nordholm, Senior Member, IEEE, Siow Yong Low, Member, IEEE, and \\ Antonio Cantoni, Fellow, IEEE
}

\begin{abstract}
A method that significantly improves the convergence rate of the gradient-based blind signal separation (BSS) algorithm for convolutive mixtures is proposed. The proposed approach is based on the steepest descent algorithm suitable for constrained BSS problems, where the constraints are included to ease the permutation effects associated with the convolutive mixtures. In addition, the method is realized using a modified golden search method plus parabolic interpolation, and this allows the optimum step size to be determined with only a few calculations of the cost function. Evaluation of the proposed procedure in simulated environments and in a real room environment shows that the proposed method results in significantly faster convergence for the BSS when compared with a fixed step-size gradient-based algorithm. In addition, for blind signal extraction where only a main speech source is desired, a combined scheme consisting of the proposed BSS and a postprocessor, such as an adaptive noise canceller, offers impressive noise suppression levels while maintaining low-target signal distortion levels.
\end{abstract}

Index Terms-Blind signal separation (BSS), gradient based, optimization, second order, step-size search.

\section{INTRODUCTION}

$\mathbf{M}$ ICROPHONE arrays have been used successfully for speech enhancement in various applications including personal digital assistants (PDAs) and mobile phones. This is due to the fact that microphone arrays provide spatial diversity, which can be exploited to give spatial selectivity (e.g., [1]-[3]).

Microphone array techniques can be largely classified into two broad areas-namely beamforming and blind signal separation (BSS). Both approaches share the commonality of filtering and combining the microphone signals to best extract the

Manuscript received February 21, 2006; revised September 18, 2006. This work was supported by the National ICT Australia (NICTA), which is funded through the Australian Government's Backing Australia's Ability initiative, and in part by the Australian Research Council. The associate editor coordinating the review of this manuscript and approving it for publication was Dr. A. Rahim Leyman.

H. H. Dam is with the Western Australian Telecommunications Research Institute (WATRI) and Department of Mathematics and Statistics, Curtin University of Technology, Perth 6102, Australia (e-mail: dam@watri.org.au).

S. Nordholm is with the Western Australian Telecommunications Research Institute (WATRI), Curtin University of Technology, Perth 6102, Australia (e-mail: sven@watri.org.au).

S. Y. Low was with the Western Australian Telecommunications Research Institute (WATRI), Perth 6102, Australia. He is now with Sensear Pty. Ltd., Perth 6009, Australia (e-mail: siowyong.low@ sensear.com).

A. Cantoni is with the Western Australian Telecommunications Research Institute (WATRI), The University of Western Australia, Perth 6009, Australia (e-mail: cantoni@watri.org.au).

Color versions of one or more of the figures in this paper are available online at http://ieeexplore.ieee.org.

Digital Object Identifier 10.1109/TSP.2007.894406 source(s) of interest. Traditional beamforming methods require information, such as array geometry and source localization, to form a beam toward the source of interest. In the second case, all of the sources are separated from their mixtures without $a$ priori knowledge of the sources or the arrays. Currently, BSS is an emerging field of interest since the only assumption required is that the sources are statistically independent [4], [5]. Thus, unlike its counterpart beamforming, BSS has the ability to separate sources from the observed mixtures without source localization or array geometry information. Such flexibility has made BSS a popular technique in many applications (e.g., image enhancement, speech enhancement, and biomedical signal processing).

In general, there are two types of BSS approaches, namely, higher-order-based BSS [5]-[7] and second-order-based BSS [8]-[11]. These two approaches require different assumptions regarding the signal statistics. For example, higher-order-based BSS generally requires an assumption about the sources' density functions [12]. Second-order based BSS, on the other hand, requires assumption about the second-order statistics such as nonstationarity or nonwhiteness [10].

The slow rate of convergence is frequently an issue with gradient-based BSS, particularly for convolutive mixtures. For realtime applications, convergence and complexity are critically important as the algorithm is required to converge fast to reflect changes in statistics. In [13]-[16], adaptive step-size algorithms have been investigated for higher-order-based BSS, applicable for instantaneous mixing. The formulated optimization problems are unconstrained optimization problems. The unmixing filters and the step sizes are adaptively updated for every iteration, depending on the gradient.

In this paper, we consider the convolutive mixing problems with second-order gradient-based BSS. Additional constraints on the time-domain unmixing filters are included to ease the permutation problem associated with convolutive mixings [8]. Thus, these problems are formulated as constrained optimization problems. Consequently, the adaptive step-size methods described for instantaneous mixing are not directly applicable.

Here, we propose a scheme which is based on the steepest descent algorithm to improve the convergence characteristics of the gradient-based BSS with fixed step size, described in [8]. The proposed method is realized using a modified Golden search procedure [18] plus a parabolic interpolation to determine the optimum step size in each iteration. By using Brent's method [19], a parabola is fitted between the points where the minimum step size is located and the optimized step size can be effectively estimated with just a few calculations of the cost function. An important feature of the proposed method is that 
it does not require a priori selection of step-size parameters used in published adaptive step-size algorithms for instantaneous mixing.

As an application for speech enhancement for blind signal extraction where there is usually a single desired speech signal in a noisy environment, we combine the proposed BSS with a postprocessor, such as an adaptive noise canceller (ANC) [20], to further reduce the influence of noise in the dominant BSS speech output. In this case, the BSS is employed as a preprocessor to give a separation of the speech signal of interest and the noisy background.

Evaluations of the proposed algorithm have been performed in simulated environments and in a real room hands-free environment. Results show that faster convergence compared with fixed step-size BSS is obtained by incorporating the optimum step-size search. Apart from the improved convergence rate, it has been observed in evaluations that the proposed approach achieves a desired cost function value with fewer total number of operations than the fixed step-size algorithm described in [8].

The outline of the paper is given as follows. Second-order BSS is formulated in Section II following the approach described in [8], and the notation employed in the paper is introduced. In Section III, the proposed modification to the fixed step-size gradient-based BSS algorithm is presented. The complexity of the proposed BSS algorithm is derived in Section IV. Section V illustrates the use of the proposed algorithm for speech enhancement application with one dominant speech output, where the BSS is combined with a postprocessor, such as an ANC. Finally, Section VI presents the simulation results, and Section VII concludes the paper.

\section{BSS BLIND SignAL SEPARATION}

Consider the model used in [8] for a convolutive mixture of $N$ sources in which the received signal vector for $L$ microphones $\mathbf{x}(n)=\left[x_{1}(n), \cdots, x_{L}(n)\right]^{T}$ is

$$
\mathbf{x}(n)=\sum_{p=0}^{P-1} \mathbf{h}(p) \mathbf{s}(n-p)+\mathbf{v}(n)
$$

where $n$ is the sampled time index at the time instant $t=n T$ and $T$ denotes the sampling period. The matrix $\mathbf{h}(p) 0 \leq p \leq$ $P-1$ is a $L \times N$ mixing matrix, where $P$ is the length of the impulse responses from the sources to the microphones. The $N \times 1$ vector $\mathbf{s}(n-p)$ contains $N$ sources at the time index $n-p$

$$
\mathbf{s}(n-p)=\left[s_{1}(n-p) \cdots, s_{N}(n-p)\right]^{T}
$$

while $\mathbf{v}(n)$ is the noise and $[\cdot]^{T}$ denotes transposition. The received signal vector is passed through unmixing matrices $\mathbf{w}(q)$ with dimension $N \times L$, where $0 \leq q \leq Q-1$ and $Q$ are the length of the unmixing filters, to produce an output signal vector that can be expressed as

$$
\mathbf{y}(n)=\sum_{q=0}^{Q-1} \mathbf{w}(q) \mathbf{x}(n-q) .
$$

The objective is to find the unmixing matrices $\mathbf{w}(0), \cdots, \mathbf{w}(Q-1)$ to recover the sources (up to an arbitrary scaling and permutation) [8], [17]. The exact number of sources is usually unknown and is generally assumed to be the same as the number of microphones $N=L$. Consequently, the unmixing matrices $\mathbf{w}(q)$ and $0 \leq q \leq Q-1$ are assumed to have the dimension $L \times L$ for all $q$.

As in [8], we define the $K$-point windowed discrete Fourier transform (DFT) $\mathbf{X}\left(\omega_{k}, n\right)$ with normalized frequency $\omega_{k} \in$ $\Omega=\left\{\omega_{0}, \omega_{1}, \cdots, \omega_{K-1}\right\}$ and index $n$ as

$$
\mathbf{X}\left(\omega_{k}, n\right)=\sum_{\tau=0}^{K-1} \mathbf{x}(n K \alpha+\tau) e^{-j \omega_{k} \tau / K}
$$

where $\alpha$ is an overlapping factor and $j=\sqrt{-1}$. Since the BSS method in [8] exploits the nonstationarity of signals, the received signal is processed in block BSS. Furthermore, the processing is carried out in the frequency domain. Thus, the DFT domain received signal is divided into $M$ blocks of data. Denote $B$ as the number of samples in each block. For $0 \leq m \leq M-1$, the cross-power spectrum for the $m^{\text {th }}$ block, $\mathbf{R}_{x}\left(\omega_{k}, m\right)$, can be obtained as

$$
\mathbf{R}_{x}\left(\omega_{k}, m\right)=\frac{1}{B} \sum_{b=0}^{B-1} \mathbf{X}\left(\omega_{k}, m B+b\right) \mathbf{X}^{H}\left(\omega_{k}, m B+b\right)
$$

where $(\cdot)^{H}$ denotes Hermitian transposition. Note that the model in (1) involves linear convolution whereas frequency-domain processing using the DFT corresponds to circular convolution. Assumptions dealing with linear/circular convolution and also nonstationarity issues related to our problem are covered in [8].

The task at hand is to estimate $L \times L$ matrix $\mathbf{W}\left(\omega_{k}\right)$ for each frequency $\omega_{k}$ that jointly decorrelates all of the $M$ matrices in (4) for all $k$. As proposed in [8], this problem can be mathematically formulated as minimizing the following weighted cost function over all of the frequencies $\omega_{k}$ :

$$
f\left(\mathbf{W}\left(\omega_{0}\right), \cdots, \mathbf{W}\left(\omega_{K-1}\right)\right)=\sum_{k=0}^{K-1} \gamma\left(\omega_{k}\right) \sum_{m=0}^{M-1}\left\|\mathbf{E}\left(\omega_{k}, m\right)\right\|_{F}^{2}
$$

where the matrix $\mathbf{E}\left(\omega_{k}, m\right)$ is the diagonalization error

$$
\mathbf{E}\left(\omega_{k}, m\right)=\mathbf{W}\left(\omega_{k}\right) \mathbf{R}_{x}\left(\omega_{k}, m\right) \mathbf{W}^{H}\left(\omega_{k}\right)-\Lambda_{s}\left(\omega_{k}, m\right)
$$

and $\|\cdot\|_{F}^{2}$ is the Frobenius norm.

The matrix $\Lambda_{s}\left(\omega_{k}, m\right)$ is a diagonal matrix with elements equal to $\mathbf{W}\left(\omega_{k}\right) \mathbf{R}_{x}\left(\omega_{k}, m\right) \mathbf{W}^{H}\left(\omega_{k}\right)$ along the diagonal. Thus, for all $0 \leq m \leq M-1$ and $0 \leq l_{1}, l_{2} \leq L-1$, we have

$$
\begin{array}{r}
{\left[\Lambda_{s}\left(\omega_{k}, m\right)\right]_{l_{1}, l_{2}}} \\
\quad= \begin{cases}{\left[\mathbf{W}\left(\omega_{k}\right) \mathbf{R}_{x}\left(\omega_{k}, m\right) \mathbf{W}^{H}\left(\omega_{k}\right)\right]_{l_{1}, l_{2}},} & \text { if } l_{1}=l_{2} \\
0, & \text { if } l_{1} \neq l_{2}\end{cases}
\end{array}
$$

where $[\cdot]_{l_{1}, l_{2}}$ denotes the $\left(l_{1}, l_{2}\right)$ element of a matrix [.]. The weighting function $\gamma\left(\omega_{k}\right)$ is obtained based on the correlation matrices $\mathbf{R}_{x}\left(\omega_{k}, m\right), 0 \leq m \leq M-1$, as

$$
\gamma\left(\omega_{k}\right)=\frac{1}{\sum_{m=0}^{M-1}\left\|\mathbf{R}_{x}\left(\omega_{k}, m\right)\right\|_{F}^{2}} .
$$


The minimization of the weighted cost function in (5) can lead to an arbitrary permutation of the frequency bins. A method to overcome this problem is to constrain the corresponding timedomain unmixing weight $\mathbf{w}(\tau)$ of $\mathbf{W}\left(\omega_{0}\right), \cdots, \mathbf{W}\left(\omega_{K-1}\right)$ [8] as

$$
\mathbf{w}(\tau)=\mathbf{0}_{L \times L} \quad \forall \tau \geq D
$$

where $D \ll K$ and $\mathbf{0}_{L \times L}$ are a $L \times L$ zero matrix. We now reformulate the equivalent constraints to (7) in terms of the frequency-domain unmixing matrices. Denoting $\mathbf{G}$ and $\mathbf{P}$, respectively, as the $K$-point inverse DFT matrix operation and a $(K-D) \times K$ matrix

$$
\mathbf{P}=\left[\mathbf{0}_{(K-D) \times D}, \mathbf{I}_{(K-D) \times(K-D)}\right]
$$

where $\mathbf{0}_{(K-D) \times D}$ and $\mathbf{I}_{(K-D) \times(K-D)}$ are a $(K-D) \times D$ zero matrix and a $(K-D) \times(K-D)$ identity matrix, respectively. The constraint (7) can be reduced to

$$
\mathbf{P w}_{l_{1}, l_{2}}=\mathbf{0}_{(K-D) \times 1} \quad \forall 0 \leq l_{1}, l_{2} \leq L-1
$$

where $\mathbf{w}_{l_{1}, l_{2}}=\left[[\mathbf{w}(0)]_{l_{1}, l_{2}}, \cdots,[\mathbf{w}(K-1)]_{l_{1}, l_{2}}\right]^{T}$ and $[\mathbf{w}(\tau)]_{l_{1}, l_{2}}, 0 \leq \tau \leq K-1$, is the $\left(l_{1}, l_{2}\right)^{\text {th }}$ element of matrix $\mathbf{w}(\tau)$. The vector $\mathbf{0}_{(K-D) \times 1}$ is a $(K-D) \times 1$ zero vector. The constraints in (9) are equivalent to the following linear constraints on the frequency-domain unmixing matrices:

$$
\mathbf{P G W}_{l_{1}, l_{2}}=\mathbf{0}_{(K-D) \times 1} \quad \forall 0 \leq l_{1}, l_{2} \leq L-1
$$

where

$$
\mathbf{W}_{l_{1}, l_{2}}=\left[\left[\mathbf{W}\left(\omega_{0}\right)\right]_{l_{1}, l_{2}}, \cdots,\left[\mathbf{W}\left(\omega_{K-1}\right)\right]_{l_{1}, l_{2}}\right]^{T}
$$

with $\left[\mathbf{W}\left(\omega_{k}\right)\right]_{l_{1}, l_{2}}$ denoting the $\left(l_{1}, l_{2}\right)^{\text {th }}$ element of the matrix $\mathbf{W}\left(\omega_{k}\right)$.

To avoid the degenerate solution of matrices with zero entries, it is necessary to introduce additional constraints on the elements of the unmixing matrices. One possible constraint is to restrict the diagonal elements of $\mathbf{W}\left(\omega_{k}\right)$ to be one for all $k$. Alternatively, the unmixing matrices can be forced to be unitary [10], [17]. These constraints on the unmixing matrices can be incorporated as constraints in the optimization problem or included as penalty functions in a weighted cost [17].

The problem of minimizing (5) with (10) and additional constraints on the diagonal of the unmixing matrices can be viewed as an optimization problem over $\left[\mathbf{W}\left(\omega_{k}\right)\right]_{l_{1}, l_{2}}, l_{1} \neq l_{2}$, with diagonal elements of $\mathbf{W}\left(\omega_{k}\right)$ chosen as constant

$$
\left[\mathbf{W}\left(\omega_{k}\right)\right]_{l_{1}, l_{2}}=1 \quad \forall l_{1}=l_{2}, 0 \leq k \leq K-1 .
$$

It follows from (11) that along the diagonal, (10) is satisfied for all $l_{1}=l_{2}$. Thus, the optimization problem can be formulated as (12), shown at the bottom of the page.

\section{STEEPEST DESCENT AlgORITHM AND GRADIENT DIRECTION}

The problem (12) is a constrained optimization problem with linear constraints. By reducing it to an unconstrained optimization problem [21], this problem can be solved by using the Newton method. However, as (12) is optimized over all unmixing matrices $\mathbf{W}\left(\omega_{k}\right), \forall 0 \leq k \leq K-1$, the number of reduced variables is still large. Thus, the Newton method applied to this problem has high computational complexity. Hence, the gradient approach is employed in this case for the optimization problem.

In this section, a two-level adaptive optimization procedure, which includes a step-size optimization, is developed for solving the problem (12) using the gradient search. In general, the convergence of the gradient search algorithm depends on the value of the step size $\mu$. The gradient-based algorithms converge slowly for small values of $\mu$ and may diverge for large values of $\mu$. Thus, it is desirable to obtain the best value of $\mu$ for each iteration.

\section{A. Gradient Direction}

As the first step, we derive the gradient direction of the cost function at the $i$ th iteration, where $i$ is an integer. The partial derivative of the cost function $f\left(\mathbf{W}^{(i)}\left(\omega_{0}\right), \cdots, \mathbf{W}^{(i)}\left(\omega_{K-1}\right)\right)$ at the $i$ th iteration with respect to the unmixing weights $\mathbf{W}^{(i) *}\left(\omega_{k}\right), 0 \leq k \leq K-1$, can be expressed as

$$
\begin{aligned}
& {\left[\nabla f\left(\mathbf{W}^{(i)}\left(\omega_{k}\right)\right)\right]_{l_{1}, l_{2}}} \\
& \triangleq\left[\frac{\partial f\left(\mathbf{W}^{(i)}\left(\omega_{0}\right), \cdots, \mathbf{W}^{(i)}\left(\omega_{K-1}\right)\right)}{\partial \mathbf{W}^{(i) *}\left(\omega_{k}\right)}\right]_{l_{1}, l_{2}} \\
& = \begin{cases}{\left[2 \gamma\left(\omega_{k}\right) \sum_{m=0}^{M-1} \mathbf{E}^{(i)}\left(\omega_{k}, m\right)\right.} & \\
\left.\times \mathbf{W}^{(i)}\left(\omega_{k}\right) \mathbf{R}_{x}\left(\omega_{k}, m\right)\right]_{l_{1}, l_{2},} & \text { if } l_{1} \neq l_{2} \\
0, & \text { if } l_{1}=l_{2}\end{cases}
\end{aligned}
$$

where

$\mathbf{E}^{(i)}\left(\omega_{k}, m\right)=\mathbf{W}^{(i)}\left(\omega_{k}\right) \mathbf{R}_{x}\left(\omega_{k}, m\right) \mathbf{W}^{(i) H}\left(\omega_{k}\right)-\Lambda_{s}^{(i)}\left(\omega_{k}, m\right)$.

Equivalently, we have

$$
\begin{aligned}
& \nabla f\left(\mathbf{W}^{(i)}\left(\omega_{k}\right)\right) \\
& =\text { off }\left[2 \gamma\left(\omega_{k}\right) \sum_{m=0}^{M-1} \mathbf{E}^{(i)}\left(\omega_{k}, m\right) \mathbf{W}^{(i)}\left(\omega_{k}\right) \mathbf{R}_{x}\left(\omega_{k}, m\right)\right]
\end{aligned}
$$

where "off[.]" is a matrix with elements equal to [.] outside the diagonal and zero along the diagonal.

$$
\left\{\begin{array}{l}
\min _{\left[\mathbf{W}\left(\omega_{k}\right)\right]_{l_{1}, l_{2}}, l_{1} \neq l_{2}, 0 \leq k \leq K-1} f\left(\mathbf{W}\left(\omega_{0}\right), \cdots, \mathbf{W}\left(\omega_{K-1}\right)\right) \\
\mathbf{P G} \mathbf{W}_{l_{1}, l_{2}}=\mathbf{0}_{(K-D) \times 1}, \quad \forall 0 \leq l_{1}, l_{2} \leq L-1, l_{1} \neq l_{2} \\
\text { where }\left[\mathbf{W}\left(\omega_{k}\right)\right]_{l_{1}, l_{1}} \text { is a constant, }\left[\mathbf{W}\left(\omega_{k}\right)\right]_{l_{1}, l_{1}}=1, \quad \forall k, \text { and } 0 \leq l_{1} \leq L-1 .
\end{array}\right.
$$




\section{B. Step-Size Search for the Gradient Descent Algorithm in Each Iteration}

We now develop a procedure to search for an optimum step size for each iteration $i$. Denote $\mathcal{P}\{\cdot\}$ as the projection operator for a set of matrices into the space defined by the linear constraints in (12). The optimum step size $\mu^{(i)}$ is obtained so that

$$
\begin{array}{r}
\mu^{(i)}=\arg \min _{\mu>0} f\left(\mathcal { P } \left\{\mathbf{W}^{(i)}\left(\omega_{k}\right)-\mu \nabla f\left(\mathbf{W}^{(i)}\left(\omega_{k}\right)\right),\right.\right. \\
0 \leq k \leq K-1\}) .
\end{array}
$$

The search for $\mu^{(i)}$ can be viewed as a one-dimensional optimization problem with respect to $\mu$. Thus, a line search method that utilizes the idea of the golden search algorithm for obtaining the minimum in one interval [18] is modified for suitability to this problem. Since the line search requires the calculation of the cost function a number of times, with each time requiring the projection of the unmixing matrices into the space defined by linear constraints in (12), the number of projections can be reduced by 1) projecting the gradient direction (15) into the space defined by the linear constraints in (12) to obtain a projected direction

$$
\begin{aligned}
&\left\{\hat{\nabla} f\left(\mathbf{W}^{(i)}\left(\omega_{k}\right)\right), 0 \leq k \leq K-1\right\} \\
& \quad=\mathcal{P}\left\{\nabla f\left(\mathbf{W}^{(i)}\left(\omega_{k}\right)\right), 0 \leq k \leq K-1\right\} .
\end{aligned}
$$

2) searching for an optimum step size in this direction. Since

$$
\left\{\mathbf{W}^{(i)}\left(\omega_{k}\right), 0 \leq k \leq K-1\right\}=\mathcal{P}\left\{\mathbf{W}^{(i)}\left(\omega_{k}\right), 0 \leq k \leq K-1\right\}
$$

the problem (16) reduces to

$$
\begin{aligned}
& \mu^{(i)}=\arg \min _{\mu>0} f( \mathbf{W}^{(i)}\left(\omega_{k}\right)-\mu \hat{\nabla} f( \\
&\left.\mathbf{W}^{(i)}\left(\omega_{k}\right)\right), \\
&0 \leq k \leq K-1)
\end{aligned}
$$

and 3) once the optimum step size is obtained, the coefficient update can be projected into the space defined by the linear constraints to prevent the accumulation of numerical errors.

For notational convenience, we define a one-dimensional function $g(\mu)$ with respect to the parameter $\mu$ as

$$
g(\mu)=f\left(\mathbf{W}^{(i)}\left(\omega_{k}\right)-\mu \hat{\nabla} f\left(\mathbf{W}^{(i)}\left(\omega_{k}\right)\right), 0 \leq k \leq K-1\right) .
$$

The search for an optimum step size can be described as follows.

Procedure III.1: Search for an optimum step size $\mu^{(i)}$ that minimizes the cost function (19).

Step 1) Initialize a step size $\mu>0$, a constant $\delta>1$, and an accuracy level $\epsilon_{1}$. Set $\mu_{0}=0, s=\mu$, and $\mu_{1}=s$.

Step 2) Obtain the cost functions $g\left(\mu_{0}\right)$ and $g\left(\mu_{1}\right)$. If $g\left(\mu_{0}\right) \leq g\left(\mu_{1}\right)$, then reduce the initial step size by setting $s / \delta \rightarrow s$. Let $\mu_{1}=s$ and go to the beginning of Step 2. Otherwise, $g\left(\mu_{0}\right)>g\left(\mu_{1}\right)$ and continue to Step 3).

Step 3) Increase $s$ by setting $\delta s \rightarrow s$ and let $\mu_{2}=\mu_{1}+$ $s$. Calculate the cost function $g\left(\mu_{2}\right)$. If $g\left(\mu_{1}\right)>$ $g\left(\mu_{2}\right)$, then set $\mu_{0}=\mu_{1}, \mu_{1}=\mu_{2}$ and return to the beginning of Step 3). Otherwise, go to Step 4).
Step 4) We have three points $\mu_{0}, \mu_{1}$, and $\mu_{2}$, satisfying

$$
g\left(\mu_{0}\right)>g\left(\mu_{1}\right), g\left(\mu_{1}\right) \leq g\left(\mu_{2}\right) \text { and } \mu_{0}<\mu_{1}<\mu_{2}
$$

Thus, there exists a local minimum in the interval $\left[\mu_{0}, \mu_{2}\right]$. Brent's method using parabolic interpolation [19] is then employed to search for the local optimum step size in the interval $\left[\mu_{0}, \mu_{2}\right]$. Following that, a parabola is fitted among three points $\left[\mu_{0}, g\left(\mu_{0}\right)\right],\left[\mu_{1}, g\left(\mu_{1}\right)\right]$, and $\left[\mu_{2}, g\left(\mu_{2}\right)\right]$. The minimum of this parabola in the interval $\left[\mu_{0}, \mu_{2}\right]$ can be calculated as

$$
\begin{aligned}
a & =\mu_{1}-\frac{1}{2} \\
& \times \frac{\left(\mu_{1}-\mu_{0}\right)^{2}\left[g\left(\mu_{1}\right)-g\left(\mu_{2}\right)\right]-\left(\mu_{1}-\mu_{2}\right)^{2}\left[g\left(\mu_{1}\right)-g\left(\mu_{0}\right)\right]}{\left(\mu_{1}-\mu_{0}\right)\left[g\left(\mu_{1}\right)-g\left(\mu_{2}\right)\right]-\left(\mu_{1}-\mu_{2}\right)\left[g\left(\mu_{1}\right)-g\left(\mu_{0}\right)\right]}
\end{aligned}
$$

with the corresponding cost function $g(a)$. Next, progress to Step 5).

Step 5) If $\left|g(a)-g\left(\mu_{1}\right)\right|<\epsilon_{1}$, then go to Step 6). Otherwise, we have one of the following two cases.

Case 1) The value of $a$ falls in the interval $\left[\mu_{0}, \mu_{1}\right]$. If $g(a)<g\left(\mu_{1}\right)$, then set $\mu_{2}=\mu_{1}, \mu_{1}=$ $a, g\left(\mu_{2}\right)=g\left(\mu_{1}\right)$, and $g\left(\mu_{1}\right)=g(a)$. Otherwise, set $\mu_{0}=a$ and $g\left(\mu_{0}\right)=g(a)$.

Case 2) The value of $a$ falls in the interval $\left[\mu_{1}, \mu_{2}\right]$. If $g(a)<g\left(\mu_{1}\right)$, then set $\mu_{0}=\mu_{1}, \mu_{1}=$ $a, g\left(\mu_{0}\right)=g\left(\mu_{1}\right)$ and $g\left(\mu_{1}\right)=g(a)$. Otherwise, set $\mu_{2}=a$ and $g\left(\mu_{2}\right)=g(a)$.

For both cases, the new set of three points $\left[\mu_{0}, g\left(\mu_{0}\right)\right],\left[\mu_{1}, g\left(\mu_{1}\right)\right]$, and $\left[\mu_{2}, g\left(\mu_{2}\right)\right]$ satisfies the constraints in (20). Thus, return to Step 4).

Step 6) If $g(a)<g\left(\mu_{1}\right)$, then let $\mu_{1}=a$. Set the optimum step size $\mu^{(i)}$ as $\mu^{(i)}=\mu_{1}$ and stop the procedure.

The advantage of Procedure III.1 is that it is relatively simple since only the cost function (19) is required to be calculated. In addition, by combining parabolic interpolation with an increasing step size search for each step by $\delta>1$, the optimum step size $\mu^{(i)}$ can be found with just a few calculations of the cost function.

\section{Summary of the Steepest Descent Gradient-Based Optimization Procedure}

The proposed two-level optimization procedure for the problem (12) is now outlined as follows.

Procedure III.2: Two-level adaptive optimization procedure.

Step 1) Initialize the iteration $i=1$ and the time-domain unmixing matrices $\mathbf{w}^{(1)}(\tau)$ as

$$
\mathbf{w}^{(1)}(\tau)= \begin{cases}\mathbf{I}_{L \times L}, & \text { if } \tau=0 \\ \mathbf{0}_{L \times L}, & \forall \tau \geq 1\end{cases}
$$

Transform $\mathbf{w}^{(1)}(\tau)$ into the frequency domain using $K$-point DFT to obtain $\mathbf{W}^{(1)}\left(\omega_{k}\right), 0 \leq k \leq K-1$. It can be seen that $\mathbf{W}^{(1)}\left(\omega_{k}\right), 0 \leq k \leq K-1$, satisfy the constraints in (12). 
Step 2) Calculate the projected search direction $\hat{\nabla} f\left(\mathbf{W}^{(i)}\left(\omega_{k}\right)\right), 0 \leq k \leq K-1$, in (17).

Step 3) Search for an optimum step size $\mu^{(i)}$ in the direction $\hat{\nabla} f\left(\mathbf{W}^{(i)}\left(\omega_{k}\right)\right), 0 \leq k \leq K-1$, according to Procedure III.1. Update the unmixing weight matrices according to

$$
\begin{aligned}
\mathbf{W}^{(i+1)}\left(\omega_{k}\right)=\mathbf{W}^{(i)}\left(\omega_{k}\right)-\mu^{(i)} \hat{\nabla} f & \left(\mathbf{W}^{(i)}\left(\omega_{k}\right)\right), \\
& \forall 0 \leq k \leq K-1 .
\end{aligned}
$$

To prevent accumulation of numerical errors, these matrices can be projected into the space defined by the linear constraints in (12).

Step 4) Calculate the cost function

$$
\begin{gathered}
f\left(\mathbf{W}^{(i+1)}(0), \cdots, \mathbf{W}^{(i+1)}(K-1)\right) \\
\text { for the }(i+1)^{t h} \text { iteration. If } \\
\mid f\left(\mathbf{W}^{(i+1)}(0), \cdots, \mathbf{W}^{(i+1)}(K-1)\right) \\
-f\left(\mathbf{W}^{(i)}(0), \cdots, \mathbf{W}^{(i)}(K-1)\right) \mid \\
<\epsilon_{2}\left|f\left(\mathbf{W}^{(i+1)}(0), \cdots, \mathbf{W}^{(i+1)}(K-1)\right)\right|
\end{gathered}
$$

where $\epsilon_{2}$ is a small tolerance and $|\cdot|$ denotes the absolute value, then go to Step 5). Otherwise, set $i+1 \rightarrow i$ and return to Step 2).

Step 5) Stop the procedure. The optimum unmixing matrices in the frequency domain are

$$
\left\{\mathbf{W}^{(i+1)}\left(\omega_{k}\right), 0 \leq k \leq K-1\right\}
$$

with the corresponding time-domain impulse responses $\mathbf{w}^{(i+1)}(\tau)$.

\section{COMPLEXITY FOR ADAPTIVE BSS ALGORITHM}

In this section, we estimate the number of complex multiplications required in each iteration for two cases with: 1) fixed step size and 2) optimized step size. Since the observed signal is real, we only need to estimate the unmixing matrices for $K_{1}$ frequencies, where $K_{1}=K / 2+1$. To reduce the computational complexity, the first summation of the cost function in (5) is now defined only between 0 and $K / 2$. As can be seen from Algorithms III.1 and III.2, the main calculating steps required for the BSS algorithm include 1) obtaining the cost function (5); 2) calculating the gradient direction (15); and 3) obtaining the projected gradient direction.

We start with estimating the number of complex multiplications required for (5). The number of multiplications required for (6) for each $\omega_{k}$ is $2 L^{3}$. Thus, the number of multiplications required for (5) is

$$
\xi_{1}=K_{1}\left[1+M\left(2 L^{3}+L^{2}\right)\right] .
$$

Moreover, the number of multiplications required to obtain the gradient direction (15) for each iteration can be given as

$$
\xi_{2}=K_{1}\left(1+4 M L^{3}\right) .
$$

The projected gradient, on the other hand, requires two $K$-point DFT. If $K$ is chosen as a power of two, then the fast Fourier transform (FFT) can be used. Thus, a $K$-point FFT requires $K / 2 \log _{2} K$ multiplications. Consequently, the number of multiplications required to constrain the gradient is

$$
\xi_{3}=L^{2} K \log _{2} K=2 L^{2} K_{1} \log _{2}\left(2 K_{1}\right) .
$$

Hence, the number of multiplications for each iteration of the BSS with fixed step size is

$$
\nu_{1}=\xi_{2}+\xi_{3}
$$

Denote $r$ as the average number of cost function calculation required to obtain the optimized step size. The number of multiplications required in each iteration for the BSS with the optimized step size is

$$
\nu_{2}=r \xi_{1}+\xi_{2}+\xi_{3}
$$

Denote $I_{1}$ and $I_{2}$ as the number of iterations required by BSS with a fixed and optimized step size, respectively, for convergence. The complexity ratio between the optimized and the fixed step size for convergence is defined as

$$
C R=\frac{I_{2} \nu_{2}}{I_{1} \nu_{1}} \text {. }
$$

In the simulation result section, the BSS will be shown with an optimized step size and does not require as many iterations for convergence as with the fixed step size.

\section{BSS POSTPROCESSOR With ADAPTIVE NOISE CANCELLER}

As an application for speech enhancement where there is usually a single desired speech signal in a noisy environment, the improved BSS is combined with postprocessing such as an ANC [20] to further suppress the noise component in the BSS speech-dominant output. Prior to the postprocessing stage, the BSS outputs must be correctly channeled into the desired and the reference signals. Thus, we employ the kurtosis, which is a quantitative measure of non-Gaussianity of a signal, for each BSS output in order to determine the desired speech signal.

For $0 \leq l \leq L-1$, the kurtosis for the $l$ th BSS output can be estimated as shown in (29), at the bottom of the next page, where $Y_{l}\left(\omega_{k}, n\right)$ is the $l$ th element of $\mathbf{Y}\left(\omega_{k}, n\right)$, which is the output of $\mathbf{X}\left(\omega_{k}, n\right)$ through the BSS. In addition, $\sigma_{Y_{l}\left(\omega_{k}\right)}^{2}$ is the variance of $Y_{l}\left(\omega_{k}, n\right)$ and $E\{\cdot\}$ denotes the expectation over $n$.

A smaller value of kurtosis indicates that the distribution tends toward Gaussian while a higher value of kurtosis indicates that the distribution tends toward super-Gaussian. Since a speech signal has a Laplacian distribution [22], it belongs to the super-Gaussian case. In addition, it follows from the central limit theorem that the interference/noise may be due to many nondirectional sources (e.g., babble noise tends to be a Gaussian-like distribution).

By denoting $d$ as the index of the output corresponds to the largest kurtosis

$$
d=\arg \max _{0 \leq l \leq L-1} \mathcal{K}_{l}
$$


then $Y_{d}\left(\omega_{k}, n\right)$ is the speech-dominant output while $Y_{l}\left(\omega_{k}, n\right)$, $l \neq d$, are the noise-dominant outputs.

The ANC is then employed to further cancel any components correlating to $Y_{l}\left(\omega_{k}, n\right), l \neq d$, from $Y_{d}\left(\omega_{k}, n\right)$. Denote $\mathbf{c}_{l}^{(n)}\left(\omega_{k}\right)$ as the coefficient vector of the ANC filter with order $\kappa$ for the $l$ th BSS output at the $n$th iteration

$$
\mathbf{c}_{l}^{(n)}\left(\omega_{k}\right)=\left[c_{l, 1}^{(n)}\left(\omega_{k}\right), \cdots, c_{l, \kappa}^{(n)}\left(\omega_{k}\right)\right]^{T}, l \neq d .
$$

The ANC speech output is given by

$$
z\left(\omega_{k}, n\right)=y_{d}\left(\omega_{k}, n\right)-\sum_{l=0, l \neq d}^{L-1} \mathbf{c}_{l}^{(n) H}\left(\omega_{k}\right) \Xi_{l}\left(\omega_{k}, n\right)
$$

where $\Xi_{l}\left(\omega_{k}, n\right)$ and $l \neq d$ is an $\kappa \times 1$ vector of the $l$ th output

$$
\Xi_{l}\left(\omega_{k}, n\right)=\left[Y_{l}\left(\omega_{k}, n\right), \cdots, Y_{l}\left(\omega_{k}, n-\kappa+1\right)\right]^{T} .
$$

The modified frequency-domain leaky LMS algorithm [23] is used to update the ANC coefficient weight with

$\mathbf{c}_{l}^{(n+1)}\left(\omega_{k}\right)=(1-\beta) \mathbf{c}_{l}^{(n)}\left(\omega_{k}\right)+z^{*}\left(\omega_{k}, n\right) \Xi_{l}\left(\omega_{k}, n\right) f\left(\omega_{k}, n\right)$

where $f\left(\omega_{k}, n\right)$ is a nonlinear function given by

$$
f\left(\omega_{k}, n\right)=\frac{\gamma}{\kappa \sigma_{z}^{2}\left(\omega_{k}, n\right)+\gamma \sum_{l=0, l \neq d}^{L-1}\left\|\Xi_{l}\left(\omega_{k}, n\right)\right\|^{2}} .
$$

The constants $\beta$ and $\gamma$ are the leaky factor and the step size for the ANC, respectively, while $\|\cdot\|$ denotes the norm of a vector. The value $\sigma_{z}^{2}\left(\omega_{k}, n\right)$ can be obtained iteratively as

$$
\sigma_{z}^{2}\left(\omega_{k}, n+1\right)=(1-\lambda) \sigma_{z}^{2}\left(\omega_{k}, n\right)+\lambda\left|z\left(\omega_{k}, n\right)\right|^{2}
$$

where $\lambda$ is the smoothing factor.

\section{Simulation Results}

Simulations are performed with $T=512, D=128$, and $M=4$. The values of $s$ and $\delta$ are chosen as $s=1$ and $\delta=3$. The overlapping factor $\alpha$ is $\alpha=25 \%$. The tolerances $\epsilon_{1}$ and $\epsilon_{2}$ are chosen as $\epsilon_{1}=10^{-4}$ and $\epsilon_{2}=2 \times 10^{-4}$. The fixed step size and the initial step size for Procedure III.1 are chosen as $\mu=1$ [8].

\section{A. BSS in a Simulated Environment With Two Speech Sources and Two Microphones}

The sources include male and a female speech having the same power and operating at the same time. The mixing signals have 8-s duration with the sampling rate of $8 \mathrm{kHz}$.

The mixing channels are chosen to be the same as those in [17]. The impulse responses of the first set of mixing channel A are

$$
\begin{aligned}
& \mathbf{H}_{1,1}(z)=1+1.0 z^{-1}-0.75 z^{-2} \\
& \mathbf{H}_{1,2}(z)=0.5 e^{-5}+0.3 z^{-6}+0.2 z^{-7} \\
& \mathbf{H}_{2,1}(z)=-0.7 z^{-5}-0.3 z^{-6}-0.2 z^{-7} \\
& \mathbf{H}_{2,2}(z)=0.8-0.1 z^{-1}
\end{aligned}
$$
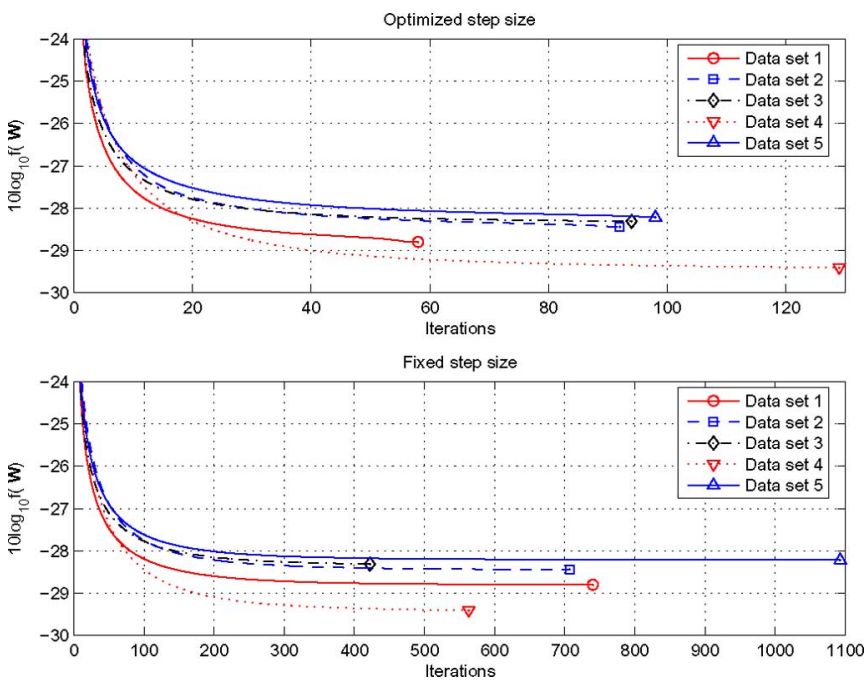

Fig. 1. Case 1: Convergence comparison for BSS with fixed and optimized step size in a simulated environment with channel A. Note that the number of iterations on the $x$-axis for the two subplots are different.

while the impulse responses of the second set of mixing channel $\mathrm{B}$ are

$$
\begin{aligned}
\mathbf{H}_{1,1}(z)= & 1+0.8 z^{-1}-0.75 z^{-2}+0.5 z^{-3}-0.4 z^{-4} \\
& -0.3 z^{-5}+0.2 z^{-6}+0.15 z^{-7}-0.07 e^{-8} \\
\mathbf{H}_{1,2}(z)= & 0.45+0.5 z^{-1}-0.4 e^{-2}+0.4 z^{-3}-0.3 z^{-4} \\
& -0.2 z^{-5}+0.3 z^{-6}-0.15 z^{-7}+0.1 e^{-8} \\
\mathbf{H}_{2,1}(z)= & 0.4-0.45 z^{-1}+0.4 z^{-2}-0.3 z^{-3}-0.2 z^{-4} \\
& +0.3 z^{-5}-0.25 z^{-6}-0.1 z^{-7} \\
\mathbf{H}_{2,2}(z)= & 1-0.7 z^{-1}+0.75 z^{-2}-0.5 z^{-3}+0.4 z^{-4} \\
& -0.35 z^{-5}+0.3 z^{-6}-0.1 z^{-1}+0.08 z^{-8}
\end{aligned}
$$

Figs. 1 and 2 plot the convergence of the BSS algorithms with fixed and optimum step size for five sets of mixing signals with the channels given in (36) and (37), respectively. The BSS with optimum step size converges when the stopping criterion in Step 4 of Procedure III. 2 is satisfied while the BSS with fixed step size runs until the cost function approaches that of the optimum step size. It can be seen that BSS with an optimum step size results in a significantly faster convergence than the one with fixed step size.

Table I shows the average signal-to-interference ratio (SIR) (in decibels) and the average distortion (in decibels) for the two speech output using both mixing channels. The BSS with the optimized step size has approximately the same SIR and distortion as with the one with a fixed step size. The BSS algorithms separate the two speech signals while maintaining low distortion of the speech signals.

Table II shows the complexity comparison between the BSS with optimized and fixed step size. The table also shows the average number of calculations required for the cost function in each step for searching the optimum step size. The BSS with an optimized step size has a lower total complexity than the one

$$
\mathcal{K}_{l}=\sum_{k=0}^{K-1} \frac{E\left\{\left|Y_{l}\left(\omega_{k}, n\right)\right|^{4}\right\}-2 E^{2}\left\{\left|Y_{l}\left(\omega_{k}, n\right)\right|^{2}\right\}-\left|E^{2}\left\{Y_{l}^{2}\left(\omega_{k}, n\right)\right\}\right|}{\sigma_{Y_{l}\left(\omega_{k}\right)}^{4}} .
$$


TABLE I

CASE 1: Second-Order BSS in Simulated Environments With Two Speech Sources ANd Two Microphones

\begin{tabular}{|c|c|c|c|c|c|}
\hline \multirow{2}{*}{$\begin{array}{c}\text { Mixing } \\
\text { channel }\end{array}$} & \multirow{2}{*}{ Step size } & \multicolumn{2}{|c|}{ Signal to interference ratio } & \multicolumn{2}{c|}{ Distortion measure } \\
\cline { 3 - 6 } & & Output 1 & Output 2 & Output 1 & Output 2 \\
\hline \multirow{2}{*}{ Channel A } & Fixed & $13.73 \mathrm{~dB}$ & $13.31 \mathrm{~dB}$ & $-18.15 \mathrm{~dB}$ & $-21.28 \mathrm{~dB}$ \\
\cline { 2 - 6 } & Optimized & $14.03 \mathrm{~dB}$ & $13.55 \mathrm{~dB}$ & $-18.16 \mathrm{~dB}$ & $-21.26 \mathrm{~dB}$ \\
\hline \multirow{2}{*}{ Channel B } & Fixed & $19.79 \mathrm{~dB}$ & $11.76 \mathrm{~dB}$ & $-20.45 \mathrm{~dB}$ & $-29.28 \mathrm{~dB}$ \\
\cline { 2 - 6 } & Optimized & $19.69 \mathrm{~dB}$ & $11.74 \mathrm{~dB}$ & $-20.43 \mathrm{~dB}$ & $-29.31 \mathrm{~dB}$ \\
\hline
\end{tabular}

TABLE II

CASE 1: COMPLEXITY COMPARISON ACCORDING TO TABLE I FOR SimUlATED ENVIRONMENTS

\begin{tabular}{|c|c|c|}
\hline $\begin{array}{c}\text { Mixing } \\
\text { channel }\end{array}$ & $\begin{array}{c}\text { Average \# of cost evaluation } \\
\text { for optimized step size }(r)\end{array}$ & $\begin{array}{c}\text { Complexity ratio (CR) in (28) } \\
\text { between optimized and fixed step size for convergence }\end{array}$ \\
\hline Channel A & 5.77 & 0.26 \\
\hline Channel B & 5.75 & 0.57 \\
\hline
\end{tabular}

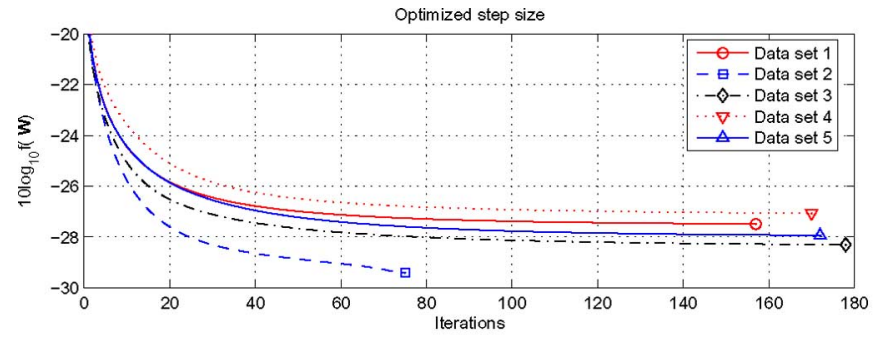

Fixed step size

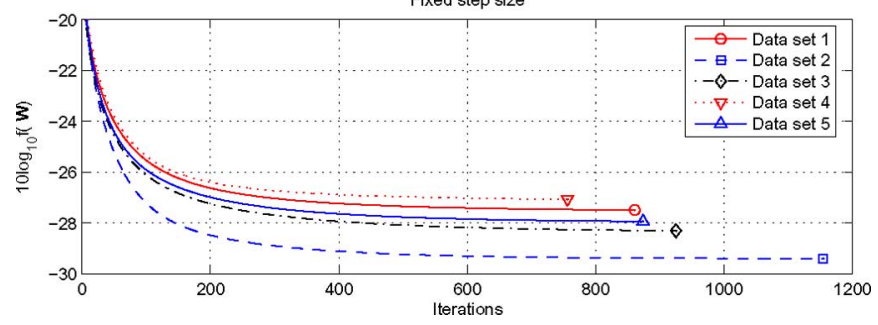

Fig. 2. Case 1: Convergence comparison for BSS with a fixed and optimized step size in a simulated environment with channel B.

with a fixed step size, even for cases when the fixed step-size algorithm converges relatively fast.

\section{B. Combined Scheme of BSS and ANC in a Real Room Environment With a Speech Source and Babble Noise}

Simulation is tested in a real room environment of dimensions $3.5 \times 3.1 \times 2.3 \mathrm{~m}^{3}$ using a linear array, sampled at $8 \mathrm{kHz}$. The interelement array distance is $0.04 \mathrm{~m}$ and the speech source is positioned $0.55 \mathrm{~m}$ at an arbitrary angle of $65^{\circ}$ from the center of the array. Similar to the first case, the algorithm is tested for five sets of a noisy received signal, each having $8 \mathrm{~s}$. The purpose of placing the source at an angle is to test whether the proposed structure is able to pick up the source without any localization information. As for the interference, two loudspeakers emitting babble noise are placed facing the front two corners of the room and three other loudspeakers (also babble) are randomly placed in the middle of the room facing the array.
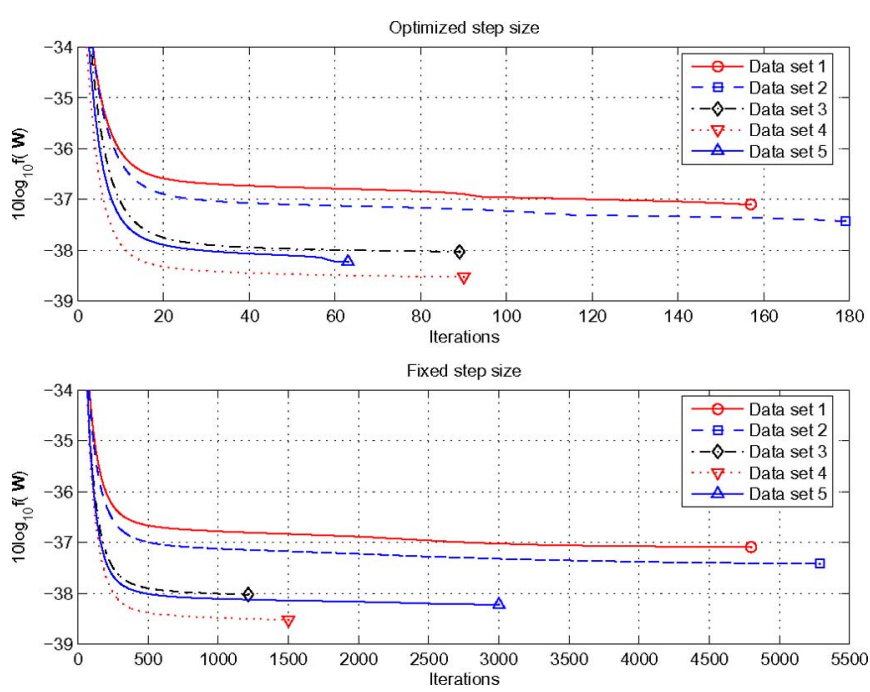

Fig. 3. Case 2: Convergence comparison for BSS with a fixed and optimized step size in a real room environment with two microphones.

Figs. 3-5 show the convergence of the BSS cost function for a fixed and optimized step size. The number of microphones increases from 2 to 4 . The BSS with an optimized step size results in a faster convergence rate than the one with a fixed step size. This is especially evident for the case with three or four microphones.

Table III shows the average signal-to-noise ratio (SNR) (in decibels) for the speech-dominant output and the noise-to-signal ratio (NSR) (in decibels) for the noise-dominant output with the number of microphones increasing from 2 to 4 . The BSS with an optimized step size has approximately the same average SNR and NSR as the one with the fixed step size.

Table IV shows the complexity comparison between the fixed and optimized step size BSS for convergence. The BSS algorithm with an optimized step size achieves overall lower com- 

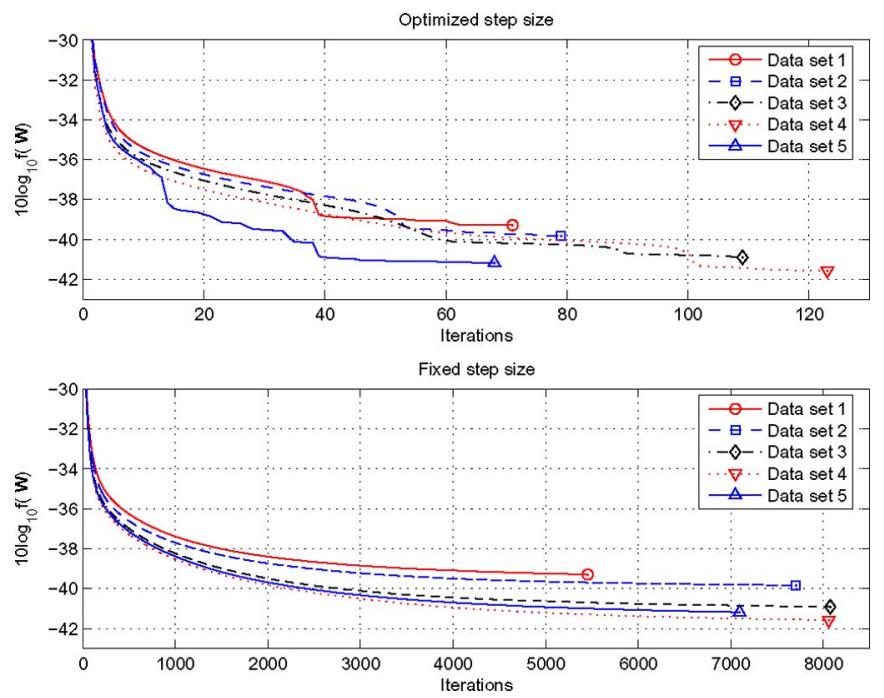

Fig. 4. Case 2: Convergence comparison for BSS with a fixed and optimized step size in a real room environment with three microphones.
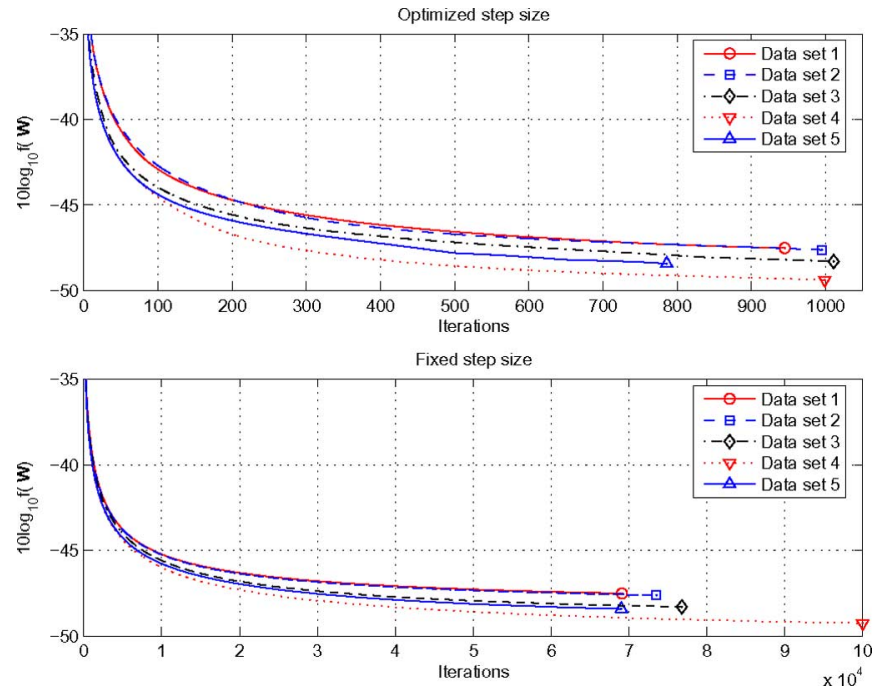

Fig. 5. Case 2: Convergence comparison for a BSS with a fixed and optimized step size in a real room environment with four microphones.

TABLE III

Case 2: Average Signal-to-Noise Ratio (SNR) For SPEech-Dominant OUTPUT AND NOISE-TO-SIGNAL RATIO (NSR) FOR NOISE-DOMINANT OUTPUT

IN A REAL RoOM ENVIRONMENT With BABBLE NoISE. THE NuMBER OF MiCROPHONES INCREASES FrOM TwO TO FOUR. THE NOISY RECEIVED SigNAL HAS AN AVERAGE SNR OF $0 \mathrm{~dB}$

\begin{tabular}{|c|c|c|c|}
\hline $\begin{array}{c}\text { Number of } \\
\text { microphones }\end{array}$ & Step size & $\begin{array}{c}\text { Speech dominant } \\
\text { output (SNR) }\end{array}$ & $\begin{array}{c}\text { Noise dominant } \\
\text { output (NSR) }\end{array}$ \\
\hline 2 & Fixed & $0.62 \mathrm{~dB}$ & $4.80 \mathrm{~dB}$ \\
\cline { 2 - 4 } & Optimized & $0.42 \mathrm{~dB}$ & $4.73 \mathrm{~dB}$ \\
\hline \multirow{2}{*}{3} & Fixed & $3.37 \mathrm{~dB}$ & $7.55 \mathrm{~dB}$ \\
\cline { 2 - 4 } & Optimized & $2.83 \mathrm{~dB}$ & $7.61 \mathrm{~dB}$ \\
\hline \multirow{2}{*}{4} & Fixed & $6.09 \mathrm{~dB}$ & $9.86 \mathrm{~dB}$ \\
\cline { 2 - 4 } & Optimized & $6.10 \mathrm{~dB}$ & $9.86 \mathrm{~dB}$ \\
\hline
\end{tabular}

TABLE IV

CASE 2: COMPLEXITY COMPARISON ACCORDING TO TABLE III FOR A REAL ROOM ENVIRONMENT WITH BABBLE NOISE

\begin{tabular}{|c|c|c|}
\hline $\begin{array}{c}\text { Number of } \\
\text { microphones }\end{array}$ & $\begin{array}{c}\text { Average \# of cost evaluation } \\
\text { for optimized step size }(r)\end{array}$ & $\begin{array}{c}\text { Complexity ratio (CR) in (28) between } \\
\text { optimized and fixed step size for convergence }\end{array}$ \\
\hline 2 & 6.83 & 0.07 \\
\hline 3 & 7.59 & 0.03 \\
\hline 4 & 8.02 & 0.04 \\
\hline
\end{tabular}
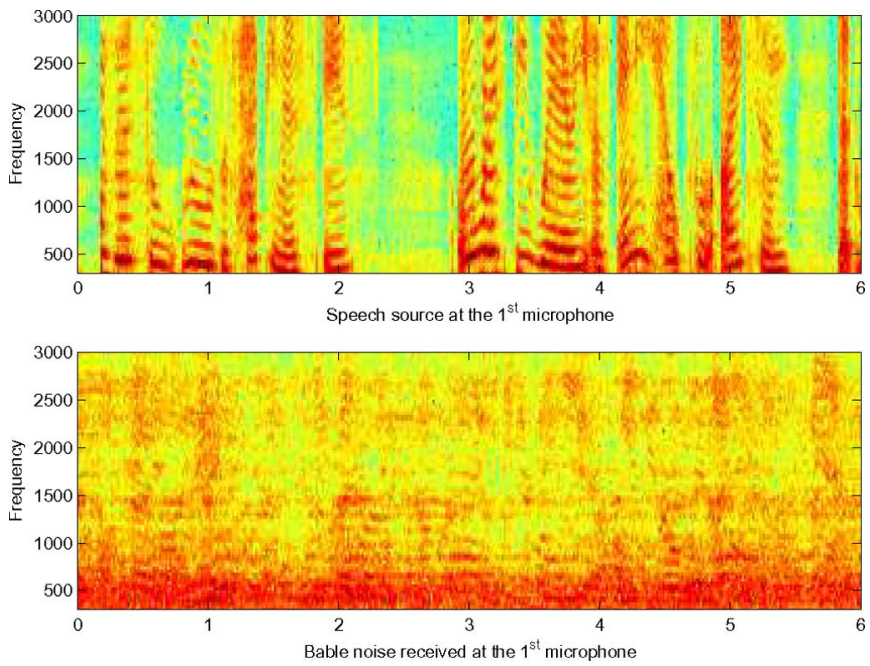

Fig. 6. Case 2: Spectrogram for the original speech and the noise component at the first microphone for the case with four microphones in a real room environment with babble noise.

plexity due to its faster convergence which results in a significant reduction in the number of iterations.

As for the combined scheme of BSS and ANC, the noise level in the speech-dominant output is reduced further by passing BSS outputs through a postprocessor, such as an ANC. The ANC exploits the temporal content of the babble-dominant output to remove the residual babble sources in the speech-dominant output. For the ANC, the parameters $\kappa, \lambda, \gamma$, and $\beta$ are chosen as $\kappa=3, \lambda=10^{-8}, \gamma=0.2$, and $\beta=0.999$.

Table V shows the SNR for the speech-dominant output of ANC during the first $4 \mathrm{~s}, 4-8 \mathrm{~s}, 8-12 \mathrm{~s}$, and $12-16 \mathrm{~s}$. The noise suppression levels increase significantly as the time increases (e.g., between 12-16 s). In addition, the noise suppression is increased with a higher number of microphones. The table also shows the distortion level for the speech outputs. The hybrid processor yields a significant noise suppression level while maintaining a low level of target signal distortion.

Fig. 6 shows the spectrogram of the source and the noise components for one of the data sets in the noisy received signal at the first microphone for the case with four elements. The spectrograms of the speech and the noise-dominant BSS outputs with optimized step size are given in Fig. 7. Clearly, the babble noise is suppressed in the speech-dominant output and dominated in the noise-dominant output.

Fig. 8 plots the spectrogram for the combined BSS and ANC output as well as the noisy received signal during the period between 8-16 s. A significant noise suppression is obtained for the ANC output while maintaining low distortion of the speech signal. 
TABLE V

Case 2: Average Signal-to-Noise Ratio (SNR) For the SPEech Dominant OutPut After the ANC FOR A REAL ROOM ENVIRONMENT WITH BABBLE NOISE

\begin{tabular}{|c|c|c|c|c|c|}
\hline \multirow{2}{*}{$\begin{array}{c}\text { Number of } \\
\text { microphones }\end{array}$} & \multicolumn{4}{|c|}{ Signal to noise ratio (SNR) } & \multirow{2}{*}{$\begin{array}{c}\text { Average } \\
\text { distortion }\end{array}$} \\
\cline { 2 - 5 } & $\begin{array}{c}\text { The first } \\
1-4(\mathrm{~s})\end{array}$ & $\begin{array}{c}\text { Between } \\
4-8(\mathrm{~s})\end{array}$ & $\begin{array}{c}\text { Between } \\
8-12(\mathrm{~s})\end{array}$ & $\begin{array}{c}\text { Between } \\
12-16(\mathrm{~s})\end{array}$ & \\
\hline 2 & $3.34 \mathrm{~dB}$ & $6.00 \mathrm{~dB}$ & $6.03 \mathrm{~dB}$ & $7.58 \mathrm{~dB}$ & $-23.01 \mathrm{~dB}$ \\
\hline 3 & $9.37 \mathrm{~dB}$ & $14.99 \mathrm{~dB}$ & $15.75 \mathrm{~dB}$ & $18.14 \mathrm{~dB}$ & $-18.76 \mathrm{~dB}$ \\
\hline 4 & $10.90 \mathrm{~dB}$ & $16.74 \mathrm{~dB}$ & $16.78 \mathrm{~dB}$ & $20.32 \mathrm{~dB}$ & $-20.74 \mathrm{~dB}$ \\
\hline
\end{tabular}
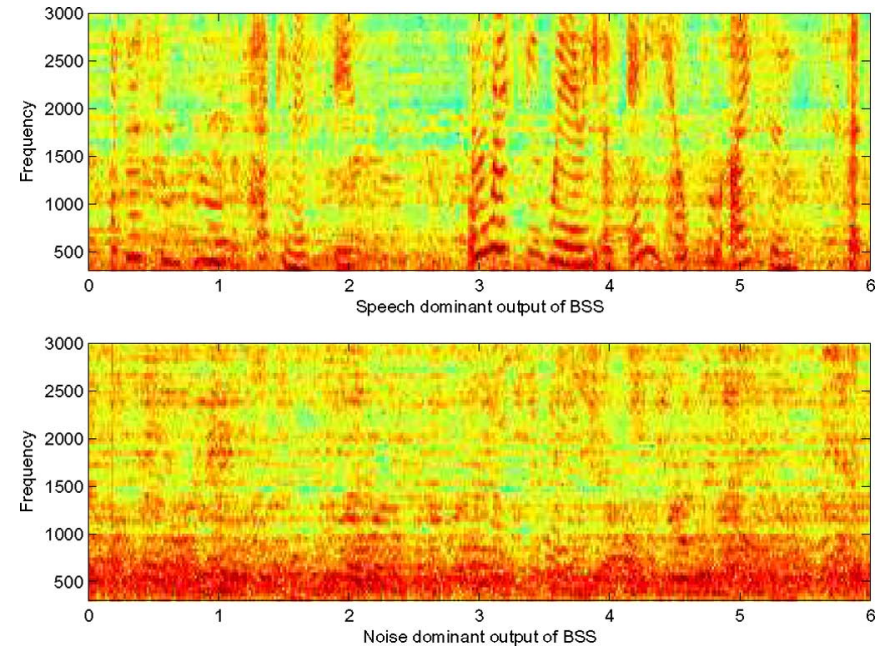

Fig. 7. Case 2: Spectrogram of the speech-dominant and the noise-dominant BSS outputs for the case with four microphones and optimized step-size BSS in a real room environment with babble noise.
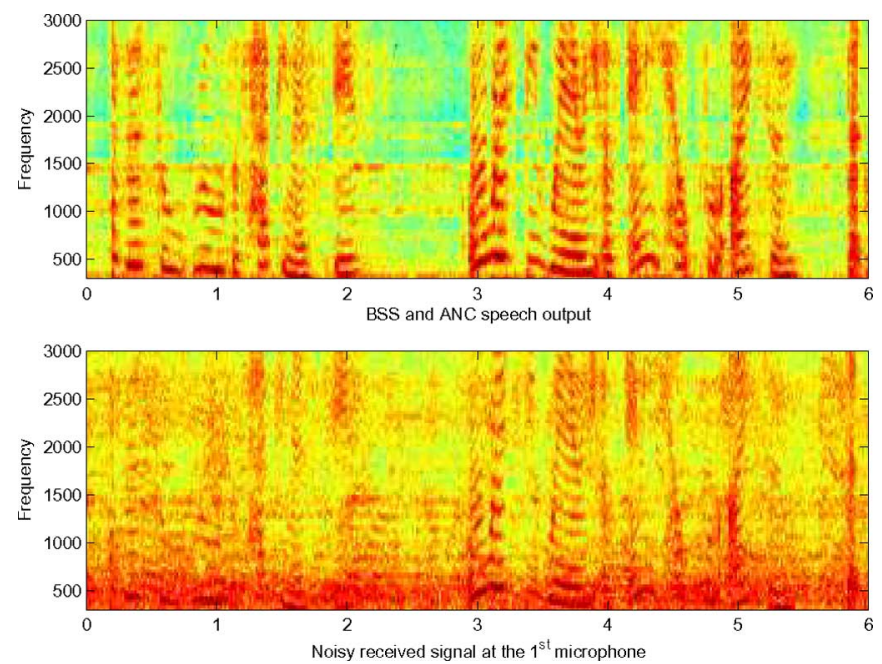

Fig. 8. Case 2: Spectrogram of the combined BSS and ANC output, and the noisy received signal for the case with four microphones and optimized step size BSS in a real room environment with babble noise.

\section{CONCLUSION}

In this paper, we have proposed a steepest descent adaptive optimization procedure which includes a step size search in the context of gradient-based second-order BSS to improve convergence and reduce complexity associated with the fixed step size. The method is realized by using a modified golden search method and a parabolic interpolation to search for an optimal step size in each iteration. The performance of the proposed algorithm evaluated in simulated environments and a real room environment shows that the algorithm results in a significantly faster convergence and with lower overall complexity when compared to the constant step-size gradient-based algorithm.

\section{REFERENCES}

[1] M. Brandstein and D. Ward, Microphone Arrays: Signal Processing Techniques and Applications, ser. Digital Signal Processing. Berlin, Germany: Springer-Verlag, 2001.

[2] J. Benesty, S. Makino, and J. Chen, Speech Enhancement. Berlin, Germany: Springer-Verlag, 2005.

[3] D. H. Johnson and D. E. Dudgeon, Array Signal Processing: Concepts and Applications. Englewood Cliffs, NJ: Prentice-Hall, 1993.

[4] P. Smaragdis, "Efficient blind separation of convolved sound mixtures," presented at the IEEE Workshop Applications Signal Process. Audio Acoust., Oct. 1997.

[5] A. Hyvärinen, J. Karhunen, and E. Oja, Independent Component Analysis. New York: Wiley, 2001.

[6] S. Haykin, Unsupervised Adaptive Filtering. New York: Wiley, 2000, vol. 1, Blind Source Separation.

[7] A. Bell and T. Sejnowski, "An information maximization approach to blind separation and blind deconvolution," Neural Comput., vol. 7, pp. 1129-1159, Nov. 1995.

[8] L. Parra and C. Spence, "Convolutive blind separation for non-stationary sources," IEEE Trans. Speech Audio Process., vol. 6, no. 3, pp. 320-327, May 2000.

[9] E. Weinstein, M. Feder, and A. V. Oppenheim, "Multi-channel signal separation by decorrelation," IEEE Trans. Speech Audio Process., vol. 1, no. 4, pp. 405-413, Oct. 1993.

[10] D. W. E. Schobben and P. W. Sommen, "A frequency domain blind signal separation method based on decorrelation," IEEE Trans. Signal Process., vol. 50, no. 8, pp. 1855-1865, Aug. 2002.

[11] H. Buchner, R. Aichner, and W. Kellermann, "A generalization of blind source separation algorithms for convolutive mixtures based on second-order statistics," IEEE Trans. Speech Audio Process., vol. 13, no. 1, pp. 120-134, Jan. 2005.

[12] N. Grbic, X.-J. Tao, S. Nordholm, and I. Claesson, "Blind signal separation using overcomplete subband representation," IEEE Trans. Speech Audio Process., vol. 9, no. 5, pp. 524-533, Jul. 2001.

[13] L. Yuan, W. Wang, and J. A. Chambers, "Variable step-size sign natural gradient algorithm for sequential blind source separation," IEEE Signal Process. Lett., vol. 12, no. 8, pp. 589-592, Aug. 2005.

[14] J. A. Chambers, M. G. Jafari, and S. McLaughlin, "Variable step-size EASI algorithm for sequential blind source separation," Electron. Lett., vol. 40, no. 6, pp. 393-394, Mar. 2004.

[15] S. C. Douglas and A. Cichocki, "Adaptive step size techniques for decorrelation and blind source separation," in Proc. Conf. Rec. 32nd Asilomar Conf. Signals, Systems and Computers, Nov. 1998, vol. 2, pp. 1191-1195.

[16] J.-F. Cardoso and B. H. Laheld, "Equivariant adaptive source separation," IEEE Trans. Signal Process., vol. 44, no. 12, pp. 3017-3030, Dec. 1996. 
[17] W. Wang, S. Sanei, and J. A. Chambers, "Penalty function-based joint diagonalization approach for convolutive blind separation of nonstationary sources," IEEE Trans. Signal Process., vol. 53, no. 5, pp. 1654-1669, May 2005.

[18] M. Minoux, Mathematical Programming Theory and Applications. New York: Wiley, 1986.

[19] R. P. Brent, Algorithms for Minimization Without Derivatives. New York: Dover, 2002.

[20] S. Y. Low, S. Nordholm, and R. Togneri, "Convolutive blind signal separation with post-processing," IEEE Trans. Speech Audio Process., vol. 12 , no. 5 , pp. 539-548, Sep. 2004.

[21] S. Boyd and L. Vandenberghe, Convex Optimization. Cambridge, U.K.: Cambridge Univ. Press, 2004.

[22] S. Gazor and W. Zhang, "Speech probability distribution," IEEE Signal Process. Lett., vol. 10, no. 7, pp. 204-207, Jul. 2003.

[23] J. E. Greenberg, "Modified LMS algorithms for speech processing with an adaptive noise canceller," IEEE Trans. Speech Audio Process., vol. 6, no. 4, pp. 338-350, Jun. 1998.

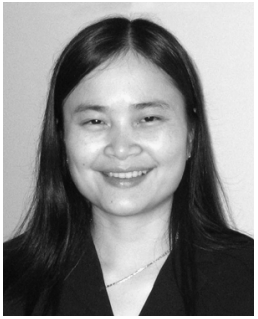

Hai Huyen Dam received the Bachelor's degree (Hons.) and the Ph.D. degree (Hons.) from Curtin University of Technology, Perth, Australia, in 1996 and 2001, respectively.

From 1999 to 2000, she was a Visiting Research Associate, spending one year at the Blekinge Institute of Technology, Karlskrona, Sweden. Currently, she is a Senior Lecturer with a joint appointment between Western Australian Telecommunications Research Institute (WATRI) and the Department of Mathematics and Statistics, Curtin University of Technology, Australia. Her research interests are adaptive array processing, optimization, equalization, and filter design.

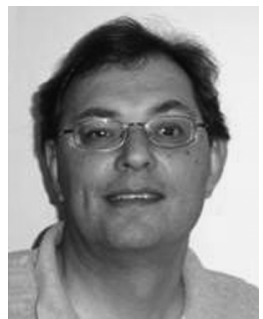

Sven Nordholm (M'91-SM'04) received the Dipl.Eng. and Ph.D. degrees from Lund University, Lund, Sweden, in 1983 and 1992, respectively. From 1983 to 1986, he was a Development Engineer with GAMBRO, Gambro, Sweden. He began his academic career at Lund University and subsequently cofounded the Department of Signal Processing at Blekinge Institute of Technology, Karlskrona, Sweden.

He was appointed Professor and Director of the Australian Telecommunications Research Institute, Perth, Australia, in 1999. Currently, he is the Research Director for Signal Processing Laboratories in the Western Australian Telecommunications Research Institute (WATRI). His research interests are adaptive array processing, optimization methods, blind signal separation, equalization, and filter design. He holds several patents and has published many publications.

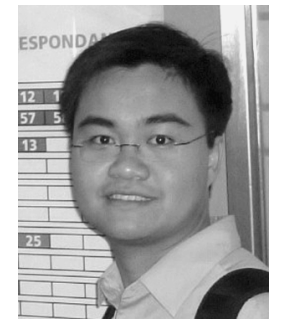

Siow Yong Low (S'99-M'05) received the B.E. and $\mathrm{Ph} . \mathrm{D}$. degrees from Curtin University of Technology, Perth, Australia, in 2001 and 2005, respectively.

He was a Research Fellow with WATRI, a joint institute between Curtin University and the University of Western Australia, Nedlands, from 2005 to 2006. Since 2007, he has been an R\&D Engineer with Sensear Pty. Ltd., Perth, Australia. His research interests include array and statistical signal processing with applications to speech enhancement.

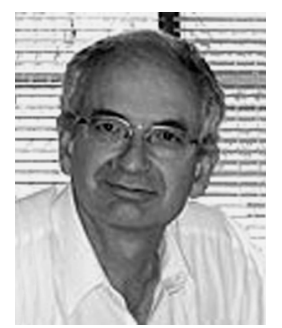

Antonio Cantoni (F'98) was born in Soliera, Italy, on October 30, 1946. He received the B.E. (Hons.) and the Ph.D. degrees from the University of Western Australia, Nedlands, in 1968 and 1972, respectively.

$\mathrm{He}$ was a Lecturer in Computer Science at the Australian National University, Canberra, in 1972. $\mathrm{He}$ joined the Department of Electrical and Electronic Engineering at the University of Newcastle, Shortland, Australia, in 1973, where he held the Chair of Computer Engineering until 1986. In 1987, he joined QPSX Communications Ltd, Perth, Australia, as Director of the Digital and Computer Systems Design Section for the development of the DQDB Metropolitan Area Network. From 1987 to 1990 , he was a Visiting Professor in the Department of Electrical and Electronic Engineering at the University of Western Australia. From 1992 to 1997, he was the Director of the Australian Telecommunications Research Institute and Professor of Telecommunications at Curtin University of Technology. During this period, he was the Director of the Cooperative Research Centre for Broadband Telecommunications and Networking. Currently, he is Chief Technology Officer with Atmosphere Networks, Inc., and Professor of Telecommunications at the University of Western Australia. He is interested in adaptive signal processing, electronic system design, and networking, and regularly acts as a Consultant to industry in these areas.

Dr. Cantoni is a Fellow of the Australian Academy of Technological Sciences and Engineering. He has been an Associate Editor of the IEEE TRANSACTIONS ON SIGNAL PROCESSING. 